\title{
Minimización del impacto ambiental en la ejecución de fachadas mediante el empleo de materiales reciclados
}

\section{Environmental impact minimization of building façades by means of recycled material usage}

M. Marrero $^{(*)}$, L. Martínez-Escobar ${ }^{(*)}$, M.P. Mercader ${ }^{(*)}$, C. Leiva ${ }^{(*)}$

RESUMEN

Nuevos materiales de construcción reciclados, desarrollados por los autores entre otros, han sido propuestos para minimizar el impacto ambiental y económico de las fachadas. Para medir dicha minimización se ha empleado un modelo de cuantificación de recursos consumidos en construcción basado en la clasificación sistemática de la medición y del presupuesto de obras de edificación del Banco de Costes de la Construcción de Andalucía. Se ha analizado la fachada de una vivienda compuesta principalmente por fábrica de ladrillo y trasdosado de paneles de yeso laminado, materiales comúnmente empleados en las viviendas en Andalucía, que controlan tanto el impacto ambiental como el económico de la solución y han sido sustituidos por paneles reciclados de yeso y placas recicladas de hormigón. Finalmente se ha comprobado mediante el modelo de cuantificación como se reduce significativamente el impacto ambiental sin incrementar el económico, quedando demostrada la sensibilidad del modelo.

\section{SUMMARY}

New recycled construction materials, developed by the authors among others, have been proposed in order to reduce the environmental and economical impact of the building facades. The model "Quantification of resources consumed in construction", by means of the bill of quantities which is based on the Andalusia Building Cost System, assesses the impact minimization. A real dwelling facade is analyzed, which is made of brick cladding and plasterboard, materials widely used in Andalusia, Spain. In the analysis these construction materials control the impact and are replaced by gypsum and concrete recycled panels, respectively. Finally the changes are evaluated by the quantification model, showing a significant reduction in the environmental and economical impact of the facade construction. The present work shows that the model "Quantification of resources consumed in construction" is sensible to changes in the construction materials.
Palabras clave: Construcción sostenible; materiales reciclados; impacto medioambiental; impacto económico; fachada.
Keywords: Sustainable construction; recycled materials; environmental impact; economical impact; facade.

\footnotetext{
(*) Universidad de Sevilla, (España).

Persona de contacto/Corresponding author: madelyn@us.es (M. Marrero).
}

Recibido/Received: 12 jul 2011 Aceptado/Accepted: 24 may 2012 Publicado online/ Published online: 14 jan 2013 


\section{INTRODUCCIÓN}

En la Unión Europea, la construcción de edificios consume el $40 \%$ de los recursos naturales y la energía primaria, y genera una proporción similar de residuos. Estos datos nos hablan de un sector profundamente impactante sobre el medio económico, ecológico y social, en definitiva un sector insostenible (1). Asimismo, los edificios, una vez construidos, continúan siendo una causa directa de contaminación por las emisiones que se producen en los mismos o su impacto sobre el territorio.

En el caso de los materiales de construcción, los costes ecológicos mayores se deben al consumo de los recursos naturales, al coste energético y a la generación de los residuos. El consumo a gran escala de determinados materiales puede llevar a su agotamiento y transformación del medio (1). Por otro lado, el material fuertemente manipulado y que ha sido sometido a un proceso de fabricación energéticamente intenso tiene unos grandes efectos medioambientales, puesto que gran cantidad de energía primaria es consumida. Emplear materiales de baja energía incorporada en todo su ciclo de vida será uno de los mejores indicadores de sostenibilidad.

Otra etapa de los materiales de construcción con un alto impacto ambiental tiene lugar en la valorización o depósito de residuos de construcción y demolición (RCD) en vertederos. Tras la demolición o desconstrucción de la edificación, un alto porcentaje de los materiales se convierten en residuos (2). Su destino, ya sea la reutilización directa, el reciclaje o la deposición en vertedero, cambia el nivel de impacto. En relación con el tratamiento de residuos, es necesario destacar el Real Decreto 105/2008, de 1 de febrero, que tiene por objeto establecer el régimen jurídico de la producción y gestión de los residuos de la construcción y demolición, con el fin de fomentar, por este orden, su prevención, reutilización, reciclado y otras formas de valoración, asegurando que los destinos a operaciones de eliminación reciban un tratamiento adecuado, y contribuir a un desarrollo sostenible de la actividad de construcción (3). Aún con la entrada en vigor del anterior decreto, la mayor parte de los RCD se llevan a vertederos, creando de esta forma un gran impacto visual y paisajístico, además de un impacto ecológico negativo al rechazar materias primas que con un adecuado tratamiento podrían ser recicladas. Se hace por tanto necesario aprovechar el potencial que tiene estos materiales como material secundario (2).
Para reducir el impacto de los RCD y también reducir la extracción de materias primas, en la mayoría de los casos recursos no renovables, surge el empleo de materiales reciclados en los nuevos productos de construcción. En la actualidad, en el sector de la edificación se percibe la proliferación de nuevos productos de construcción que incorporan materiales reciclados (pétreos, plásticos, madera, vidrio, papel, etc.), gestándose lo que se ha venido a denominar una nueva generación de eco-productos que aúnan eficiencia y compromiso medioambiental (4).

La reducción de los impactos medioambientales antes mencionados, energía, materias primas y residuos, hace necesaria una cuantificación que permita verificar el cambio de modelo por uno más sostenible. En el caso del asociado al uso de la energía primaria en la fabricación de los materiales y en la puesta en obra de los mismos, éste se expresa generalmente en megajulios (MJ) de energía embebida en el material y la cuantificación de los distintos gases emitidos a la atmósfera en kilogramos de emisiones de $\mathrm{CO}_{2}$ equivalentes producidas (5). Las herramientas que analizan dichos impactos generalmente siguen la metodología de análisis de ciclo de vida, LCA (6), o el indicador huella ecológica que se refiere a la extensión de tierra que sería necesaria para suministrar los recursos (cereales, pienso, leña, pescado y terreno urbano) y absorber las emisiones $\left(\mathrm{CO}_{2}\right)$ de la sociedad mundial, y además puede ser usado conjuntamente con otros indicadores, como la huella de carbono o la huella hídrica (7).

Una herramienta que mide los impactos antes descritos, y que está enfocada desde la perspectiva de la obra de edificación, es el modelo de cuantificación de recursos consumidos en construcción (MCRC) (8). Este modelo, descrito en detalle más adelante, parte de la medición y presupuesto del proyecto, siguiendo la estructura jerarquizada del Banco de Costes de la Construcción de Andalucía (BCCA) (9). El BCCA permite tener en cuenta todos los factores y recursos intervinientes en el proyecto: materiales, medios y mano de obra.

En esta línea se desarrollan los objetivos del presente trabajo:

- Evaluar el impacto ambiental y económico que se produce en el proceso de ejecución de una fachada, desde la óptica de los recursos materiales consumidos en su ejecución, coste energético, emisiones de $\mathrm{CO}_{2}$ producidas y residuos generados. 
- Identificar los materiales de mayor impacto en la solución constructiva estudiada.

- Cuantificar, utilizando para ello el MCRC, la minimización del impacto ambiental y económico producido en las fachadas estudiadas a través de la sustitución de los materiales de mayor impacto por nuevos materiales reciclados desarrollados por los autores entre otros, y que consisten en paneles de yeso fabricados a partir de cenizas (10), así como prefabricados de hormigón realizados con residuos de la construcción.

\section{MATERIALES}

Las fachadas, además de la función estética, deben satisfacer otros requisitos: deben ser impermeables al agua, y aislar el interior, térmica y acústicamente. La sección tipo de una fachada convencional se compone de dos hojas: una exterior, normalmente de ladrillo, y otra interior, que puede ser de ladrillo o de otros materiales como el yeso laminado. Entre esas dos hojas se coloca un aislante térmico, para lo que usualmente se utilizan materiales como el poliuretano, la fibra de vidrio o la lana de roca. Para evitar que el agua penetre a través del cerramiento se coloca un embarrado de mortero de cemento que actúa como impermeabilización, que no siempre es necesario si le transferimos esta función a un adecuado revestimiento continuo en el exterior; pero dado que en los proyectos estudiados aparece en la fachada convencional así será planteado gráficamente. Por último, es necesaria una pequeña separación de uno o dos centímetros para permitir que ventile el vapor de agua y no empape el aislamiento.

Se han elegido para el análisis los siguientes materiales de construcción por estar entre los más utilizados en las viviendas de Andalucía (8):

- Fábrica de $1 / 2$ pie de ladrillo cerámico perforado tomados con mortero de cemento M-5.

- Embarrado de Mortero de cemento CS III W1.

- Poliuretano proyectado.

- Perfiles metálicos de sustentación de la hoja interior.

- Yeso laminado.

Y para la solución alternativa de fachada se emplean materiales reciclados: paneles reciclados de yeso (10) y placas recicladas de hormigón.

El panel reciclado de yeso ha sido desarroIlado a partir de cenizas volantes producidas durante la combustión de biomasa en centrales térmicas. En este caso, la biomasa es orujillo, residuo que se genera durante la extracción de aceite a la aceituna. Estas cenizas, no son aprovechables en cementos, y se producen cada vez en mayor cantidad, debido a que la biomasa trata de sustituir al carbón, y así disminuir las emisiones de $\mathrm{CO}_{2}$. El panel reciclado de yeso está formado, además de por estas cenizas, por aglomerante y aditivos (11). Se trata de nuevo material de construcción elaborado a partir de un mortero compuesto por un alto porcentaje de residuos (>50\%p), que presenta una baja densidad $\left(<900 \mathrm{~kg} / \mathrm{m}^{3}\right)$, con un sencillo método de fabricación, una alta resistencia al fuego (conferido por la alta proporción de cenizas de biomasa en su composición) y una resistencia a compresión superior a $1 \mathrm{MPa}$. El material comparado con otros comercializados tiene prestaciones similares (10).

Los paneles de yeso laminado reciclado se fabricaron con un $60 \%$ p de cenizas volantes sin tratamiento previo para eliminar in-quemados y con vermiculita sin moler como aditivo. Como material aglomerante se ha dispuesto yeso, por aportar mayor capacidad aislante manteniendo unas propiedades mecánicas aceptables. Para satisfacer que el material cumpliera una resistencia a compresión superior a $1 \mathrm{MPa}$ se aportaron fibras, una parte en forma de fibra monofilamento de polipropileno que mejora su resistencia a compresión y otra parte en forma de malla de fibra de vidrio que desarrolla la resistencia a flexión e impacto. La composición definitiva de las placas está resuelta mediante ceniza $(60 \%)$, yeso $(30 \%)$, vermiculita $(9,5 \%)$ y fibra $(0,5 \%)(11)$.

El método de fabricación consiste en la mezcla simple de los componentes en una hormigonera hasta la obtención de una mezcla homogénea, a continuación se añade agua y se mueve nuevamente hasta la obtención de la pasta homogénea (relación agua/sólido $=0,5$ ). Una vez obtenida la pasta que se vierte sobre los moldes de encofrado, el desencofrado se realiza a las 24 horas y se cura a temperatura ambiente (temperatura media: $20^{\circ}$ y humedad relativa media: $45^{\circ}$ durante 28 días) (11).

El segundo nuevo material, la placa de hormigón reciclado, se obtiene al sustituir el árido natural por árido reciclado obtenido de una planta de tratamiento de RCD. La planta genera dos fracciones de residuos de demolición, una de árido fino y otra de árido grueso. El hormigón ha sido fabricado mediante la sustitución total de la arena tradicional por el fino (50\%p), mientras que la grava ha sido sustituida totalmente por el árido grueso (30\%p), por lo que el porcentaje por peso de residuos en el material final es del $80 \%$, y el restante $20 \%$ es cemento. 
1. Fachada convencional y con materiales reciclados.
El método de fabricación consiste en el mismo de un hormigón tradicional, se mezclan los componentes en una hormigonera, hasta la obtención de una mezcla homogénea, con una relación agua/sólidos $=0,38$. Una vez obtenido la masa se vierte, desencofrándose a las 24 horas y se cura a temperatura ambiente (temperatura media: $20^{\circ} \mathrm{C}$ y una humedad relativa de $100 \%$ ) durante 28 días.

En la Tabla 1 se muestran los resultados obtenidos en el laboratorio en el hormigón con áridos naturales y el hormigón reciclado en el cual todos sus áridos son sustituidos por RCD. La densidad a 28 días es inferior en los paneles hechos de residuos y el contenido de humedad $\mathrm{H}$, es significativamente mayor. La estabilidad volumétrica es inferior en ambos casos a $10 \mathrm{~mm}$, lo cual es exigido en los estándares europeos para otros materiales de construcción fabricados con residuos (12). Por otro lado, tanto la resistencia a compresión como a flexión disminuye en el reciclado en las muestras estudiadas. La conductividad térmica a temperatura ambiente del hormigón reciclado es $0,66 \mathrm{Wm}^{-1} \mathrm{~K}^{-1}$ siendo inferior a la de hormigones de alta densidad $\left(1,63 \mathrm{Wm}^{-1} \mathrm{~K}^{-1}\right)$ pero algo superior a los de densidad media $\left(0,51 \mathrm{Wm}^{-1} \mathrm{~K}^{-1}\right)(13)$.

Tabla 1. Propiedades físicas y mecánicas del hormigón con áridos naturales y reciclados

\begin{tabular}{|c|c|c|c|c|c|c|}
\hline Áridos & $\begin{array}{c}\text { Densidad } \\
\left(\mathbf{k g m}^{-3}\right)\end{array}$ & $\begin{array}{c}\text { Estabilidad } \\
\text { volumétrica }(\mathbf{m m})\end{array}$ & $\begin{array}{c}\mathbf{H} \\
(\%)\end{array}$ & $\mathbf{p H}$ & $\begin{array}{c}\mathbf{R}_{\mathrm{c}} \\
(\mathbf{M P a})\end{array}$ & $\mathbf{R}_{\mathrm{f}}(\mathbf{M P a})$ \\
\hline Natural & 2088 & 1,0 & 1,8 & 11,0 & 22,7 & 5,2 \\
\hline Reciclado & 1590 & 0,2 & 4,0 & 11,3 & 20,1 & 4,5 \\
\hline
\end{tabular}

\section{METODOlOGíA}

Para desarrollar el presente trabajo se ha tomado como referencia un proyecto de ejecución de una vivienda en Sevilla, que se encuentra entre medianeras, tiene dos plantas de altura y $309,37 \mathrm{~m}^{2}$ de superficie construida. De dicho proyecto se han analizado memorias, planos y mediciones.

La fachada tiene las siguientes características: cerramiento de doble hoja con cámara de aire no ventilada y aislamiento por el

1. Revestimiento exterior continuo formado po enfoscado de mortero de cemento CS ill Wl, esp. $1^{\prime} 5 \mathrm{~cm}$.

2. Fábrica de $1 / 2$ pie de ladrillo perforado $\left(24 \times I^{\prime} 5 \times 7 \mathrm{~cm}\right)$ tomado con mortero de cemento $\mathrm{M}-5$.

3. Embarrado de mortero de cemento CS III WI, esp. $1 \mathrm{~cm}$.

4. Aislamiento térmico de poliuretano proyecta4. Aislamiento térm.
do, esp. $2^{\prime} 5 \mathrm{~cm}$.

5. Cámara de aire no ventilada, esp. $3 \mathrm{~cm}$.

6. Estructura de acero galvanizado para sustentación, esp. 4'6 cm.

7. Trasdosado de cartón-yeso, esp. $1^{\prime} 2 \mathrm{~cm}$.

8. Panel de hormigón reciclado, esp. $1^{\prime} 5 \mathrm{~cm}$, fijados con anclajes de acero inoxidable.

9. Panel reciclado de yeso, esp. $1^{\prime} 5 \mathrm{~cm}$.
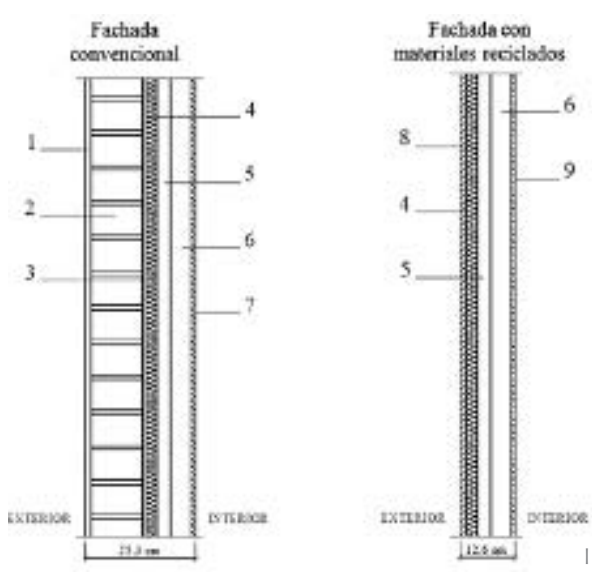

interior, compuesto por una hoja principal exterior de fábrica de ladrillo cerámico perforado, con revestimiento continuo, cámara de aire no ventilada con aislamiento térmico no hidrófilo y hoja interior de trasdosado de yeso laminado sobre estructura de acero galvanizado (Figura 1).

Se evalúan mediante el mismo procedimiento que se describe a continuación, la fachada anterior y otra solución alternativa que pretende minimizar el impacto ambiental (Figura 1).

El primer paso para evaluar el impacto ambiental de la ejecución de fachadas es analizar los materiales que la componen, ya identificados en el apartado anterior. El resto del análisis se lleva a cabo en otras dos etapas: cuantificación de los recursos materiales consumidos en su ejecución y la evaluación del impacto medioambiental de estos (8).

Para poder cuantificar los recursos consumidos, y poder compararlos, el procedimiento es el siguiente:

- Organizar las mediciones del proyecto en base a una misma estructura jerarquizada empleando la Base de Costes de la Construcción de Andalucía, BCCA (9).

- Obtener los descompuestos a través de la misma estructura (14).

- Para aquellos descompuestos que no forman parte del BCCA, ya que no son elaborados en obra, se obtiene su composición de las empresas o laboratorios especializados que los producen.

Para la evaluación del impacto ambiental, se cuantifican los siguientes indicadores por superficie construida (a partir de este momento $\mathrm{m}^{2}$ se refiere siempre a la superficie construida):

- "peso" $\left(\mathrm{kg} / \mathrm{m}^{2}\right)$

- "coste energético" $\left(\mathrm{MJ} / \mathrm{m}^{2}\right)$

- "emisiones de dióxido de carbono equivalente" (kg de $\mathrm{CO}^{2}$ eq. $/ \mathrm{m}^{2}$ )

- "RCD generados" $\left(\mathrm{kg} / \mathrm{m}^{2}\right)$

En el MCRC (12) se ha elegido la "superficie construida" como parámetro de homogenización porque el modelo evalúa no sólo las fachadas sino todos los elementos que componen el proyecto, y este parámetro permite visualizar el impacto que tiene cada elemento constructivo y compararlos con un mismo factor de ponderación en cada proyecto, finalmente, permite ver la repercusión que cada elemento constructivo tiene sobre el proyecto ejecutado. 
El indicador "peso por superficie construida" se obtiene, una vez cuantificados los materiales en las clasificaciones establecidas, aplicando el factor de relación, FR (8), que se define para cada material de construcción empleado. El factor cambia la unidad de medida del BCCA a la unidad $\mathrm{kg} / \mathrm{m}^{2}$, que se define para cada material de construcción empleado partiendo de la densidad y dimensiones de cada UD. Por ejemplo, en el caso de los ladrillos perforados se han consultado fabricantes y se define un peso medio de mil unidades de ladrillos como $2100 \mathrm{~kg}$. Las siguientes transformaciones, la "energía incorporada" $\left(\mathrm{MJ} / \mathrm{m}^{2}\right)$, las "emisiones de dióxido de carbono equivalente" ( $\mathrm{kg}$ de $\mathrm{CO}^{2}$ eq. $/ \mathrm{m}^{2}$ ) y "RCD", se definirán con respecto a este valor.

El coste energético se obtiene del indicador de la "energía incorporada"; se ha recurrido a diferentes bases de datos y publicaciones, seleccionando aquellos materiales básicos, tanto en sus materias primas como procesos de producción similares a los materiales de construcción empleados, ver Tabla 2, donde la energía incorporada se expresa en $\mathrm{MJ} / \mathrm{kg}$. Para obtener el coste energético en $\mathrm{MJ} / \mathrm{m}^{2}$ se multiplican los $\mathrm{kg} / \mathrm{m}^{2}$ de material empleados en la fachada por su respectiva energía incorporada medida en $\mathrm{MJ} / \mathrm{kg}$, valores medios de la Tabla 2.

En cuanto a las emisiones de $\mathrm{CO}_{2}$ en $\mathrm{KgCO}_{2} / \mathrm{m}^{2}$, debidas a la fabricación de los materiales, no se tienen detalles pormenorizados de las distintas fuentes energéticas y sus correspondientes proporciones consumidas en la fabricación de cada material de construcción, así que se establece que toda la energía es producida con combustibles fósiles que tienen un factor de emisiones de $0,347 \mathrm{KgCO}_{2} / \mathrm{kWh}$ (21). Una vez obtenida la energía incorporada, este se multiplica por el factor de emisiones de $\mathrm{CO}_{2}$ para combustibles fósiles y se determinan las "emisiones en $\mathrm{CO}_{2}$ " en $\mathrm{Kg} / \mathrm{m}^{2}$.

Para evaluar el impacto ambiental de la generación de residuos se emplea el modelo de cuantificación de RCD en el cual se definen un conjunto de coeficientes de transformación (22). Los coeficientes son tres: $C R$, coeficiente para medir la parte del elemento constructivo origen que se convierte en residuo; $\mathrm{CC}$, coeficiente para convertir la unidad de medida de la partida origen en la unidad de medida de la partida destino (en el MCRC es el coeficiente FR) y el CT, coeficiente para transformar el criterio de medición de la partida origen en el criterio de medición de la partida destino. Finalmente, el peso de los residuos, $\mathrm{Md}$ (o medición destino), es función del peso del los materiales, Mo (o medición origen): [1].
Tabla 2. La energía incorporada de los materiales de construcción según distintas fuentes (15) (16) (17) (18) (19) (20)

\begin{tabular}{|c|c|c|c|c|c|c|c|}
\hline Material & \multicolumn{5}{|c|}{ Energía incorporada (MJ/kg) } & \multirow{2}{*}{ Media } \\
\hline Fuentes & $(15)$ & $(16)$ & $(17)$ & $(18)$ & $(19)$ & $(20)$ & \\
\hline Ladrillo perforado & 2,85 & & & & & & 2,85 \\
\hline Poliuretano & 70 & & & 70 & 82,33 & 135 & 89,33 \\
\hline Agua & & & & & & & 0,00 \\
\hline Cemento & 7 & 7,20 & 7,20 & 7,00 & & 4,00 & 6,48 \\
\hline Árido & 0,10 & 0,10 & 0,10 & 0,10 & 0,08 & 0,50 & 0,16 \\
\hline Yeso laminado & & & & 7,90 & 5,73 & 5 & 6,21 \\
\hline Piedra & & & & 0,18 & & 0,50 & 0,34 \\
\hline Pintura & 20 & 20 & 20 & 20 & 42,23 & & 24,44 \\
\hline Árido reciclado & & & & 0,10 & & & 0,10 \\
\hline Yeso & 3,30 & 3,30 & 3,30 & 2,57 & 2,45 & 1 & 2,67 \\
\hline Vermiculita sin moler & & & & 4,53 & & & 4,53 \\
\hline Fibra de vidrio & 30 & & 30 & 22 & & 35 & 29,25 \\
\hline
\end{tabular}

[1]

$$
\mathrm{Md}=\mathrm{CR}^{*} \mathrm{CC}^{*} \mathrm{CT}^{*} \mathrm{Mo}
$$

En nuestro caso sólo se necesita determinar el coeficiente CR ya que CC es igual a FR y CT igual a 1 debido a que el criterio de medición no cambia.

\section{RESULTADOS}

\subsection{Impacto ambiental}

Una vez identificado el sistema constructivo a evaluar en el apartado 2, se ha procedido a la identificación y cuantificación de los materiales consumidos, para ello se ha partido de las mediciones en el proyecto de ejecución. Se ha realizado de forma sintética el proceso metodológico anteriormente planteado, para obtener la cuantificación del consumo de recursos en la vivienda. En la Tabla 3, la columna Qt/Sc representa la cantidad total del material consumido, expresada por la unidad de mediada empleada en el BCCA (UD) por $\mathrm{m}^{2}$ de superficie construida.

Para evaluar la generación de residuos, partiendo de la medición del proyecto, se han empleado los coeficientes de transformación, CR (22) que establecen la parte del material que se convierte en residuo, ver Tabla 3. Como cabía esperar, es el ladrillo el residuo mayoritario, al ser un material de mucho peso dentro de la fachada, representan sus residuos un $72 \%$ del peso total.

Tabla 3. Cuantificación de recursos.

\begin{tabular}{|c|c|c|c|c|c|c|c|}
\hline \multirow{2}{*}{ UD } & \multirow{2}{*}{ Material empleado } & Qt & $\mathrm{Qt} / \mathrm{Sc}$ & FR & \multirow{2}{*}{$\mathrm{kg} / \mathrm{m}^{2}$} & \multirow{2}{*}{ CR } & \multirow{2}{*}{$\begin{array}{c}\text { Residuo } \\
\left(\mathrm{Kg} / \mathrm{m}^{3}\right)\end{array}$} \\
\hline & & (UD) & $\left(\mathrm{UD} / \mathrm{m}^{2}\right)$ & (kg/UD) & & & \\
\hline $\mathrm{mu}$ & Ladrillo perforado & 5,14 & 0,02 & 2100 & 34,89 & 0,060 & 2,09 \\
\hline $\mathrm{m}^{2}$ & Poliuretano & 81,32 & 0,26 & 0,875 & 0,23 & 0,050 & 0,01 \\
\hline $\mathrm{m}^{3}$ & Agua & 1,75 & 0,01 & 1000 & 5,66 & 0,000 & 0,00 \\
\hline $\mathrm{t}$ & Cemento & 1,72 & 0,01 & 1000 & 5,56 & 0,050 & 0,28 \\
\hline $\mathrm{m}^{3}$ & Árido & 7,33 & 0,02 & 1600 & 37,91 & 0,010 & 0,38 \\
\hline $\mathrm{m}^{2}$ & Yeso laminado & 81,32 & 0,26 & 7 & 1,84 & 0,050 & 0,09 \\
\hline $\mathrm{m}^{2}$ & Piedra caliza $50 \times 50 \times 3$ & 13,50 & 0,04 & 48 & 2,09 & 0,010 & 0,02 \\
\hline $\mathrm{ml}$ & Piedra caliza 30 & 12,55 & 0,04 & 7 & 0,28 & 0,010 & 0,00 \\
\hline $\mathrm{kg}$ & Pintura acrílica & 56,63 & 0,18 & 1 & 0,18 & 0,060 & 0,01 \\
\hline \multicolumn{5}{|c|}{$\begin{array}{l}\text { TOTAL } \\
\end{array}$} & 34,89 & \multicolumn{2}{|c|}{2,89} \\
\hline
\end{tabular}


2. Comparación del coste energético por $\mathrm{m}^{2}\left(\mathrm{MJ} / \mathrm{m}^{2}\right)$
En cuanto al coste energético y las emisiones de $\mathrm{CO}_{2}$, se puede observar en la Tabla 4 que la fábrica de ladrillo cerámico genera el mayor impacto energético debido a su fabricación, requiriendo $99,44 \mathrm{MJ} / \mathrm{m}^{2}$, y por tanto un mayor impacto en relación con las emisiones, $9,58 \mathrm{CO} 2 \mathrm{Kg} / \mathrm{m}^{2}$. Siendo también, junto con el árido, el componente más pesado de la solución constructiva, lo cual representa un alto consumo de materias primas no renovables.

Una vez hecha la cuantificación del impacto, se ha analizado una solución alternativa a la convencional en el cerramiento de fachada, en la que ha tratado de sustituir los materiales de mayor impacto por materiales reciclados:

Tabla 4. Cuantificación de impacto de cada material que compone la fachada

\begin{tabular}{|c|c|c|c|c|}
\hline \multirow{2}{*}{ Material } & Peso & \multirow{2}{*}{$\begin{array}{c}\text { Energía incorporada } \\
(\mathrm{MJ} / \mathrm{kg})\end{array}$} & Energía & Emisiones \\
\hline & $\mathrm{Kg} / \mathrm{m}^{2}$ & & $\left(\mathrm{MJ} / \mathrm{m}^{2}\right)$ & $\left(\mathrm{kg} \mathrm{CO} 2 / \mathrm{m}^{2}\right)$ \\
\hline Ladrillo perforado & 34,89 & 2,85 & 99,44 & 9,58 \\
\hline Poliuretano & 0,23 & 89,33 & 20,55 & 1,98 \\
\hline Agua & 5,66 & 0 & 0,00 & 0,00 \\
\hline Cemento & 5,56 & 6,48 & 36,03 & 3,47 \\
\hline Árido & 37,91 & 0,16 & 6,07 & 0,58 \\
\hline Yeso laminado & 1,84 & 6,21 & 11,43 & 1,10 \\
\hline Piedra & 2,38 & 0,34 & 0,81 & 0,08 \\
\hline Pintura & 0,18 & 24,44 & 4,47 & 0,43 \\
\hline
\end{tabular}

Tabla 5. Cuantificación de recursos en los paneles de hormigón reciclado y de los paneles de yeso por $\mathrm{m}^{2}$ de material

\begin{tabular}{|c|c|c|}
\hline Material & Componentes & $\mathbf{k g}$ \\
\hline \multirow{3}{*}{$\begin{array}{c}\text { Paneles } \\
\text { de yeso }\end{array}$} & Cenizas de biomasa & 658,69 \\
\cline { 2 - 3 } & Yeso & 329,35 \\
\cline { 2 - 3 } & Vermiculita sin moler & 98,80 \\
\cline { 2 - 3 } & Fibra de vidrio & 5,49 \\
\cline { 2 - 3 } & Agua & 5,49 \\
\hline Total & \multicolumn{2}{|c|}{1097,82} \\
\hline \multirow{2}{*}{$\begin{array}{c}\text { Placas de } \\
\text { hormigón } \\
\text { reciclado }\end{array}$} & Cemento & 192,83 \\
\cline { 2 - 3 } & Áridos reciclado & 3159,11 \\
\hline Total & Agua & 296,00 \\
\hline
\end{tabular}

Tabla 6. Cuantificación de recursos

\begin{tabular}{|c|c|c|c|c|c|}
\hline UD & Material empleado & $\begin{array}{c}\mathbf{Q t} \\
(\mathbf{U D})\end{array}$ & $\begin{array}{c}\mathbf{Q t} / \mathbf{S c} \\
\left(\mathbf{U D} / \mathbf{m}^{\mathbf{2}}\right)\end{array}$ & $\mathbf{k g}$ & $\mathbf{k g} / \mathbf{m}^{\mathbf{2}}$ \\
\hline $\mathrm{m}^{2}$ & Hormigón reciclado & 98,89 & 0,32 & 3647,94 & 11,79 \\
\hline $\mathrm{Ud}$ & Anclajes de acero inoxidable & 198,00 & 0,64 & 5,93 & 0,02 \\
\hline $\mathrm{m}^{2}$ & Paneles de yeso reciclado & 81,32 & 0,26 & 1097,82 & 3,55 \\
\hline $\mathrm{m}$ & Montante de acero galvanizado & 25,10 & 0,08 & 34,89 & 0,11 \\
\hline
\end{tabular}

Tabla 7. Energía incorporada de los materiales de construcción según distintas fuentes

\begin{tabular}{|c|c|c|c|c|c|c|c|c|}
\hline Material & Componentes & \multicolumn{6}{|c|}{ Energía incorporada $(\mathrm{MJ} / \mathrm{kg})$} & Media \\
\hline \multirow{4}{*}{$\begin{array}{l}\text { Placas } \\
\text { de hormigón } \\
\text { reciclado }\end{array}$} & & (15) & (16) & (17) & (18) & (19) & (20) & \\
\hline & Cemento & 7 & 7,20 & 7,20 & 7,00 & & & 6,48 \\
\hline & Árido reciclado & & & & 0,10 & & & 0,10 \\
\hline & Agua & & & & & & & 0,00 \\
\hline Total & & & & & & & & 6,58 \\
\hline Anclaje & Acero inoxidable & & & & 177 & & & 177 \\
\hline Total & & & & & & & & 177 \\
\hline \multirow{5}{*}{$\begin{array}{c}\text { Paneles } \\
\text { reciclados de } \\
\text { yeso }\end{array}$} & Cenizas biomasa & & & & & & & 0,00 \\
\hline & \begin{tabular}{|l|} 
Yeso \\
\end{tabular} & 3,30 & 3,30 & 3,30 & 2,57 & 2,45 & 1 & 2,67 \\
\hline & Vermiculita & & & & 4,53 & & & 4,53 \\
\hline & Fibra de vidrio & 30 & & 30 & 22 & & 35 & 29,25 \\
\hline & Agua & & & & 0,10 & & & 0,10 \\
\hline Total & & & & & & & & 36,55 \\
\hline Montante & Acero & & & & 41,69 & & & 41,69 \\
\hline Total & & & & & & & & 41,69 \\
\hline
\end{tabular}

- Sustitución de la fábrica de ladrillo por paneles de hormigón reciclado. Las placas tienen formatos distintos según la necesidad de la obra y el diseño al que atiendan. Desde $250(\mathrm{~L}) \times 250(\mathrm{H}) \mathrm{mm}$ hasta $1800(\mathrm{~L})$ $\mathrm{x} 1900(\mathrm{H}) \mathrm{mm}$ y de espesor $1,5 \mathrm{~cm}(13)$.

- Sustitución del trasdosado del yeso laminado por paneles de yeso reciclado en cuya composición sólida encontramos cenizas, aglomerante y aditivos.

Para calcular el impacto se han desglosado en la Tabla 5 los componentes básicos que constituyen el hormigón reciclado y los paneles reciclados de yeso.

En la Tabla 6 se muestran las variaciones de los indicadores de impacto de cada material, totalizando los impactos respectivos (peso, energía y emisiones), y comparándose con las de la solución original.

Para determinar el coste energético y las emisiones de $\mathrm{CO}_{2}$, la Tabla 7 detalla la energía incorporada en los materiales según distintas fuentes bibliográficas, empleándose la media en los cálculos.

Siguiendo el mismo proceso de la fachada convencional, en la Tabla 8 se han determinado los valores totales correspondientes al coste energético y emisiones de $\mathrm{CO}_{2}$ de los materiales reciclados. Un cuarto indicador medioambiental se ha determinado, el peso de los residuos a ser generados por la fachada.

El coste energético y emisiones de $\mathrm{CO}_{2}$ de los materiales reciclados se comparan con los valores materiales convencionales en la Figura 2. Se puede apreciar que al sustituir los materiales convencionales por materiales reciclados se reducen ambos impactos. También ocurre una reducción en el porcentaje de residuos generados, en la solución original se generaban $3,1 \mathrm{~kg} / \mathrm{m}^{2}$ frente a la solución con materiales más ligeros que generan $0,208 \mathrm{~kg} / \mathrm{m}^{2}$, ya que las placas de hormigón reciclado generan menos kilogramos de residuos.

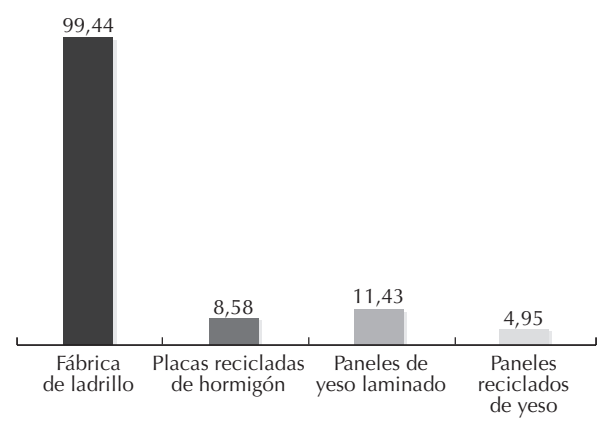




\subsection{Impacto económico}

Otro aspecto importante al hablar de sistemas más sostenibles es la evaluación del impacto económico de las nuevas propuestas. En la Tabla 9 se expone el presupuesto de ejecución de la fachada estudiada (18). El precio del " $\mathrm{m}^{2}$ Cerramiento exterior Citara+trasdosado de yeso laminado" es el precio más importante y dentro de este precio unitario se identifican los precios básicos del ladrillo y del panel de yeso laminado empleado en la vivienda (Tabla 10).

Para el cálculo del precio del panel de yeso reciclado, que no es un material que se comercialice actualmente, se ha tenido en cuenta la composición en la que se sustituye el $60 \%$ del yeso por cenizas de biomasa, por lo que los paneles de yeso son $40 \%$ de material convencional. Al sustituir el $60 \%$ del material por residuo se reduce el $60 \%$ de los costes de materia prima, y los costes de fabricación y transporte permanecen iguales, aproximadamente el coste de las materias primas es el $30 \%$ del coste total del material final, por lo que la reducción del precio es del 18\% aproximadamente.
Por otro lado en el cálculo del precio de las placas de hormigón reciclado se sigue un cálculo similar al anterior en la cual se disminuye el $80 \%$ de la materia prima, y las materias primas son un $30 \%$ del coste total del material, reduciendo el coste, con respecto a placas comercializadas de hormigón con formato similar, en un $24 \%$. No se tiene en cuenta en el análisis el coste del perfil o el de la mano de obra más intensa de la fábrica de ladrillos, sólo el coste de la materia prima. El hormigón prefabricado tiene un coste medio de 40,00 €/m² (información comercial), por lo que el coste de las placas fabricadas a partir de árido reciclado se estima en $30,40 € / \mathrm{m}^{2}$, los restantes costes, la mano de obra y el pequeño material, se definen a partir de los precios de revestimientos de fachada en el BCCA, $6,35 € / \mathrm{m}^{2}$.

En la Figura 3 se ha representado la diferencia en el coste al sustituir los materiales que producen mayor impacto ambiental por materiales reciclados donde se puede apreciar que también se reduce el impacto económico con los paneles de yeso

Tabla 8. Desglose por tipologías de cantidades de materiales de construcción usados como materiales alternativos en el sistema constructivo en fachada

\begin{tabular}{|c|c|c|c|c|c|c|c|}
\hline Material & Componentes & $\mathrm{kg} / \mathrm{m}^{2}$ & $\begin{array}{c}\text { Energía } \\
\text { incorporada }(\mathrm{MJ} / \mathrm{kg})\end{array}$ & $\begin{array}{l}\text { Energía } \\
\left(\mathrm{MJ} / \mathrm{m}^{2}\right)\end{array}$ & $\begin{array}{c}\text { Emisiones } \\
\left(\mathrm{CO} 2 \mathrm{~kg} / \mathrm{m}^{2}\right)\end{array}$ & CR & $\begin{array}{c}\text { Residuos } \\
\left(\mathrm{kg} / \mathrm{m}^{2}\right)\end{array}$ \\
\hline & Cemento & 0,62 & 6,48 & 4,02 & & 0,05 & 0,031 \\
\hline Hormigón & Áridos reciclado & 10,21 & 0,1 & 1,02 & & 0,01 & 0,102 \\
\hline \multirow[t]{2}{*}{ reciclado } & Agua & 0,96 & 0 & 0 & & 0 & 0 \\
\hline & Sub-total & & & 5,04 & 0,49 & & 0,133 \\
\hline Anclaje & Acero inoxidable & 0,02 & 177 & 3,54 & 0,34 & 0,05 & 0,001 \\
\hline Total & & & & 8,58 & 0,83 & 0 & \\
\hline \multirow{6}{*}{$\begin{array}{c}\text { Paneles } \\
\text { reciclados } \\
\text { de yeso }\end{array}$} & Cenizas biomasa & 2,13 & 0 & 0 & & 0,05 & 0 \\
\hline & Yeso & 1,06 & 2,67 & 2,83 & & 0,05 & 0,053 \\
\hline & Vermiculita & 0,32 & 4,53 & 1,54 & & 0,05 & 0,016 \\
\hline & Fibra de vidrio & 0,02 & 29,25 & 0,58 & & 0 & 0,001 \\
\hline & Agua & 0,02 & 0 & 0 & & & 0 \\
\hline & \begin{tabular}{|l|} 
Sub-total \\
\end{tabular} & & & 4,95 & 0,48 & 0,05 & 0,07 \\
\hline Montante & Acero galvanizado & 0,08 & 41,69 & 3,33 & & & 0,004 \\
\hline Total & & & & 8,28 & 0,80 & & 0,208 \\
\hline
\end{tabular}

Tabla 9. Presupuesto de ejecución material de la fachada convencional

\begin{tabular}{|l|c|c|c|}
\hline \multicolumn{1}{|c|}{ Descripción } & Cantidad & Precio(18) & Importe \\
\hline $\mathrm{m}^{2}$ Cerramiento exterior: citara y trasdosado de yeso laminado & 81,32 & 41,79 & 3398,36 \\
\hline $\mathrm{m}^{2}$ Aislamiento de capa poliuretano proyectado 2,5 cm & 81,32 & 4,60 & 374,07 \\
\hline $\mathrm{m}^{2}$ Fábrica de un pie de espesor de ladrillo perforado para revestir & 17,57 & 33,26 & 584,38 \\
\hline $\mathrm{m}^{2}$ Enfoscado maestreado/fratasado en paredes & 13,50 & 13,49 & 182,12 \\
\hline $\mathrm{m}^{2}$ Enfoscado sin /maestreado y fratasado en paredes & 102,96 & 9,93 & 1022,39 \\
\hline $\mathrm{m}^{2}$ Aplacado fachada placa caliza 3cm/50x50 & 13,50 & 54,60 & 737,10 \\
\hline $\mathrm{ml}$ Remate piedra caliza 30cm ancho & 12,55 & 36,99 & 464,22 \\
\hline $\mathrm{m}^{2}$ Pintura elastómero acrílica lisa para exteriores & 102,96 & 3,51 & 361,39 \\
\hline \multicolumn{2}{|c|}{ Total } & & 7124,03 \\
\hline
\end{tabular}

Tabla 10. Precio de los materiales que condicionan el nivel de impacto

\begin{tabular}{|l|c|c|c|}
\hline \multicolumn{1}{|c|}{ Descripción } & Cantidad & Precio & Importe \\
\hline $\mathrm{m}^{2}$ Panel de yeso laminado de $15 \mathrm{~mm}$ espesor. & 81,32 & 2,61 & 212,25 \\
\hline $\mathrm{mu}$ Ladrillo cerámico $25 \times 11,5 \times 5 \mathrm{~cm}$ & 5,14 & 77,72 & 399,48 \\
\hline $\mathrm{m}^{2}$ Panel de yeso reciclados de $15 \mathrm{~mm}$ espesor. & 81,32 & 2,14 & 174,02 \\
\hline $\mathrm{m}^{2}$ de hormigón reciclado & 17,57 & 40,00 & 702,08 \\
\hline
\end{tabular}


3. Comparación de coste $(€)$ entre materiales convencionales y materiales reciclados. reciclados pero se incrementa levemente con el uso de hormigón reciclado, siendo el coste final de la fachada prácticamente el mismo con y sin materiales reciclados, sólo se aprecia un leve incremento de 23,09 euros en el total.

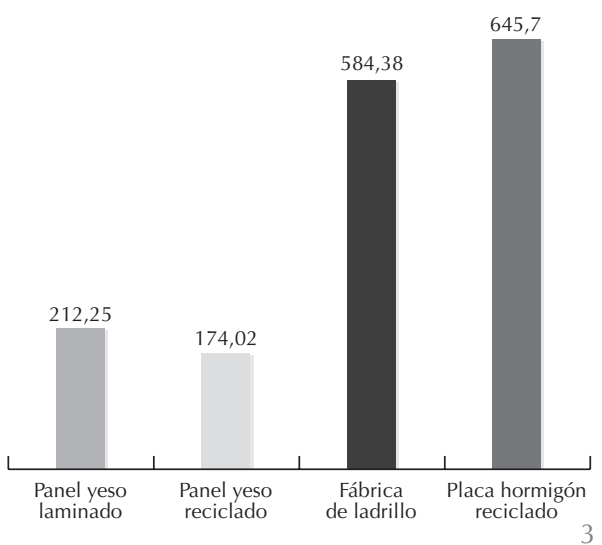

\section{CONCLUSIONES}

Un primer análisis de los resultados obtenidos permite identificar que los materiales cerámicos y los áridos son los recursos que controlan el proceso de fachada, por ejemplo, representan el $78 \%$ de todo el peso de la fachada.

Sin embargo el impacto energético y emisiones de $\mathrm{CO}_{2}$ obedecen principalmente a la cantidad de energía incorporada al material y no tanto al peso del mismo, por ello no existe paralelismo entre el peso y los otros dos indicadores (energía y emisiones). En el caso del trasdosado de yeso laminado, aunque tiene un peso reducido, requiere una gran incorporación de energía y en consecuencia importantes emisiones de $\mathrm{CO}_{2}$. Otro material que también requiere de una importante incorporación de energía es la cerámica, y en consecuencia genera grandes emisiones.
Al realizar la sustitución del panel de yeso laminado por otro panel hecho de residuos se logran significativas reducciones de energía incorporada y emisiones. Al utilizar materiales con baja energía incorporada, estaremos reduciendo proporcionalmente las emisiones de $\mathrm{CO}_{2}$, ya que los materiales analizados se ha establecido en el presente análisis que no se fabrican empleando energías limpias o renovables. En relación al peso de los residuos a ser generados por la fachada, en la solución original se generaban $3,1 \mathrm{~kg} / \mathrm{m}^{2}$ frente a la solución con materiales más ligeros que generan $0,208 \mathrm{~kg} / \mathrm{m}^{2}$, ya que se ha sustituido el ladrillo por placas de hormigón reciclado.

En cuanto al impacto en los costes, se aprecia una reducción en el coste de los paneles de yeso laminado pero un incremento en la sustitución de los ladrillos por paneles de hormigón reciclados, este incremento podría compensarse si los paneles se fabrican in situ con los propios residuos de la obra, ya que representan un ahorro en el canon de vertido de RCD.

Observando los resultados obtenidos en este estudio, se han Ilegado a disminuciones en los impactos muy significativas que permiten concluir que se pueden lograr en las etapas de extracción, producción y gestión de residuos de los materiales de construcción importantes reducciones en el consumo de materia prima y energía primaria, siendo del orden de las que se consiguen cuando se interviene en la etapa de uso y mantenimiento del edificio.

\section{AGRADECIMIENTOS}

Esta investigación se ha realizado dentro del proyecto ARCEVA, subvencionado por la Junta de Andalucía, Consejería de Vivienda y Ordenación del Territorio (Boja n. ${ }^{\circ} 94$ del 13 de mayo de 2008).

\section{REFERENCIAS}

(1) Baño Nieva, A., Vigil-Escalera Del Pozo, A. (2005). Guía de Construcción Sostenible. Edición ISTAS, Instituto Sindical de Trabajo, Ambiente y Salud. España.

(2) del Río Merino, M., Izquierdo Gracia, P., Salto Weis Azevedo, I. (2010). Sustainable construction: construction and demolition waste reconsidered. Waste Management and Research, 28(2): 118-129, doi: 10.1177/0734242X09103841.

(3) Real Decreto 105/ 2008, de 1 de Febrero, por el que se regula la producción y gestión de los residuos de construcción y demolición.

(4) Pérez Bernal, I. (2008) Eco productos: en la arquitectura y el diseño. p. 252, Barcelona, AxE-Arquitectura y entorno S.L.

(5) Argüello Méndez, T. R., Cuchí Burgos, A. (2008). Análisis del impacto ambiental asociado a los materiales de construcción empleados en las viviendas de bajo coste del programa 10 x10 con Techo-Chiapas del CYTED. Informes de la Construcción, 60(509): 25-34, doi:10.3989/ic.2008.v60.i509.588.

(6) Zabalza Bribián, I., Valero Capilla, A., Aranda Usón, A. (2011). Life cycle assessment of building materials: Comparative analysis of energy and environmental impacts and evaluation of the eco-efficiency improvement potential. Building and Environment, 46(5): 1133-1140 
(7) Galli, A., Wiedmann, T., Ercin, E., Knoblauch, D., Ewing, B., Giljum, S. (2011) Integrating Ecological, Carbon and Water footprint into a Footprint Family of indicators: Definition and role in tracking human pressure on the planet. Ecological Indicators. doi:10.1016/j.ecolind.2011.06.017.

(8) Mercader Moyano, P., Marrero, M., Solís Guzmán, J., Montes Delgado, M. V., Ramírez de Arellano, A. (2010). Cuantificación de los recursos materiales consumidos en la ejecución de la Cimentación. Informes de la Construcción, 62(517): 125-132. doi: 10.3989/ic.09.000.

(9) Base de Costes de la Construcción de Andalucía 2008. Consejería de Vivienda y Ordenación del Territorio. Junta de Andalucía. http://www.juntadeandalucia.es/viviendayordenaciondelterritorio/www/jsp/estatica.jsp?pma $=0 \& c t=-1 \& p m s a=0 \&$ e=biblioteca archivos/../planificacion/publicaciones/banco_precios_construccion/bcca08/bcca08. html Consultado: 25/03/2011.

(10) Alba, M.D., Marrero, M., Leiva, C., Montes, M.V., Vilches, L. (2012). Empleo de paneles compuestos por subproductos de centrales térmicas en fachadas trasdosadas. Informes de la Construcción, 64(526): 179-190. doi: 10.3989/ic.10.042.

(11) Leiva, C., Vilches, L. F., Vale, J., Fernández Pereira, C. (2009). Fire-Resistance of Biomass ash panels used for internal partitions in buildings. Fire Safety Journal, 44(4): 622 628. doi:10.1016/j.firesaf.2008.12.005.

(12) EN 450-1 (2005) Fly ash for concrete. Part 1: Definitions, specifications and conformity criteria.

(13) EN 993-15 (2005) Test methods for dense shaped refractory products. Part 15: Determination of thermal conductivity by hot-wire (parallel) method.

(14) Marrero, M., Ramirez-de-Arellano, A. (2010). The building cost system in Andalusia: application to construction and demolition waste management. Construction Management and Economics, 28(5): 495-507. doi:10.1080/01446191003735500.

(15) Dirección General de la Vivienda, la Arquitectura y el Urbanismo, Institut Cerdá, IDEA. Guía de la edificación Sostenible, Calidad Energética y Medio Ambiental en Edificación. Ministerio de Fomento, Madrid, España, 1999.

(16) Cuchí i Burgos, A., López Caballero, I. (1999). Informe MIES: una Aproximación al impacto Ambiental de la Escuela de Arquitectura del Vallés. Universitat Politécnica de Cataluña, Barcelona, España.

(17) Cuchí i Burgos A. (2005). Arquitectura i sostenibilitat. Universitat Politécnica de Cataluña, Barcelona, Espana.

(18) ITEC. Metabase-TCQ 2000: Datos Ambientales. ITEC, Barcelona, Cataluña, 2005.

(19) Nye M., Rydin Y. (2008). The Contribution of Ecological Footprinting to Planning Policy Development: Using REAP to Evaluate Polices for Sustainable Housing Construction. Planning and Design, 35(2): 227-247. doi:10.1068/b3379.

(20) Berge, B. (2009). The Ecology of Building Materials. Architectural Press, Amsterdam, Holanda.

(21) IDAE. Cálculo de Emisiones de $\mathrm{CO}_{2}$ de una Vivienda Estándar. IDAE. Madrid, España, 1998.

(22) Solís-Guzmán, J., Marrero, M., Montes-Delgado, M. V., Ramírez-de-Arellano, A. (2009). A Spanish model for quantification and management of construction waste. Waste Management, 29(9): 2542-2548. doi:10.1016/j.wasman.2009.05.009. 